\title{
Michael Ruck
}

\section{Die Tradition der deutschen Verwaltung}

\section{Einleitung}

„Eine einmal voll durchgeführte Bürokratie gehört zu den am schwersten zu zertrümmernden sozialen Gebilden. " ${ }^{1}$ Diese theoretische Einsicht des Soziologen Max Weber ist im Nachhinein von der deutschen Verwaltungsgeschichte des 20. Jahrhunderts ebenso eindrucksvoll bestätigt worden, wie die ironische Rückschau des Juristen Otto Mayer auf die politische Revolution von 1918/19: „Groß Neues ist ja seit 1914 und 1917 nicht nachzutragen.“ - „Verfassungsrecht vergeht, Verwaltungsrecht besteht"

Knapp ein halbes Jahrhundert später identifizierte Wolfgang Zapf die „Verwalter der Macht" als wichtige „Träger der gesellschaftlichen Kontinuitär“" über Umbrüche im Verfassungssystem und auf dem Feld der politischen Elitenrekrutierung hinweg ${ }^{3}$. Mit Blick darauf beklagte Ralf Dahrendorf gleichzeitig - Mitte der 1960er Jahre - die notorische „Seßhaftigkeit der Verwaltungselite in den Stürmen politischen Wandels" als ein wesentliches Hemmnis für die nachhaltige Durchsetzung der „liberalen Demokratie in Deutschland“4. Und 1975 konnte Theodor Eschenburg unwidersprochen feststellen: „In den drei Umbrüchen von 1918, 1933 und 1945 hatte das Berufsbeamtentum, wenn auch unter ganz unterschiedlichen Erscheinungsformen und Auswirkungen, sich mit dem ihm eigenen Beharrungsvermögen zu halten gewußt." Erst gegen Ende der 1960er Jahre, als sich ein epochaler "Generationswechsel" innerhalb der höheren Beamtenschaft vor dem Hintergrund tiefgreifender gesellschaftlich-politischer Neuorientierungen vollzogen habe, sei die säkulare "Tendenz zur Verwaltungskontinuität“ in Deutschland wenn schon nicht gebrochen, so doch merklich abgeschwächt worden ${ }^{5}$.

1 Max Weber, Wirtschaft und Gesellschaft. Grundriß der verstehenden Soziologie, hrsg. von Johannes Winckelmann, Studienausgabe (Tübingen ${ }^{5} 1976$ ) 569; vgl. ebd. 835.

2 Otto Mayer, Deutsches Verwaltungsrecht (Leipzig ${ }^{31924}$; unveränd. Nachdr. Berlin 1969) Vorwort (zuerst 1895; ${ }^{21914 / 17) . ~}$

3 Wolfgang Zapf, Die Verwalter der Macht. Materialien zum Sozialprofil der höheren Beamtenschaft, in: ders. (Hrsg.), Beiträge zur Analyse der deutschen Oberschicht (München 21965) 77-154, hier 77.

4 Ralf Dabrendorf, Gesellschaft und Demokratie in Deutschland (München 1965 u.ö.) 280 (erstes Zitat), 39 (zweites Zitat); vgl. ebd. 276, 281.

5 Theodor Eschenburg, Regierung, Bürokratie und Parteien 1945-1949. Thre Bedeutung für 
In der neueren Forschung ist diese Kontinuitätsthese bisweilen wieder in Frage gestellt worden. Gestützt auf eine qualitative und quantitative Auswertung umfangreicher Quellenstudien bestätigte indessen eine Kollektivbiographie der südwestdeutschen Verwaltungsjuristen die zwanzig Jahre alte Aussage Eschenburgs: „Wenn es im 20. Jahrhundert überhaupt einen Kontinuitätsbruch in der (südwest)deutschen Verwaltung gegeben haben sollte, dann hat er sich weder 1918 noch 1933 oder 1945 vollzogen, sondern in den späten sechziger Jahren."6

Allerdings beziehen sich diese Aussagen empirisch nur auf die Beamtenschaft als personalen Träger der Verwaltung wie als soziale Gruppe. Zu klären bleibt darüber hinaus, ob und inwieweit sie auch für die institutionelle Seite der modernen Verwaltung - normative Aufgaben, behördliche Organisation und materielle Ressourcen - allgemeine Geltung besitzen.

\section{Beharrung im Wandel der deutschen Verwaltung während des 19. und 20. Jahrhunderts}

Anfang der 1990er Jahre hat Bernd Wunder der künftigen Verwaltungs- und Beamtengeschichte die zentrale „Forschungsaufgabe“ gestellt, herauszufinden, „ob und wann Verwaltungstätigkeit restaurativ, systemstabilisierend oder modernisierend" gewesen sei’. Dieser Schlüsselfrage ist Thomas Ellwein wenig später in seiner verwaltungshistorischen Regionalstudie über Ostwestfalen-Lippe mit einem doppelten Anliegen nachgegangen: Was sagt erstens der „Zustand der Verwaltung" über den modernen Staat des 19./20. Jahrhunderts aus und was tragen zweitens die Antworten zum „Verständnis des gegenwärtigen Staates“ bei ${ }^{8}$ ? Nicht nur

die politische Entwicklung der Bundesrepublik, in: Vierteljahrshefte für Zeitgeschichte (VfZ) 24 (1976) 58-74, hier 70; vgl. ders., Der bürokratische Rückhalt, in: Die Zweite Republik. 25 Jahre Bundesrepublik Deutschland. Eine Bilanz, hrsg. von Richard Löwenthal, Hans-Peter Schwarz (Stuttgart 1974) 64-94, hier 89.

6 Michael Ruck, Korpsgeist und Staatsbewußtsein. Beamte im deutschen Südwesten 19281972 (München 1996) 266, im folgenden zitiert: Ruck, Korpsgeist; vgl. ders., Administrative Eliten in Demokratie und Diktatur. Beamtenkarrieren in Baden und Württemberg von den zwanziger Jahren bis in die Nachkriegszeit, in: Regionale Eliten zwischen Diktatur und Demokratie. Baden und Württemberg 1930 bis 1952, hrsg. von. Cornelia Rauh-Kühne, Michael Ruck (München 1993) 37-69, hier 61-63.

7 Bernd Wunder, Zur Geschichte der deutschen Beamtenschaft. Literaturbericht 1945-1985, in: Geschichte und Gesellschaft (GG) 17 (1991) 256-277. Eine souveräne Zusammenfassung des seinerzeitigen Forschungsstandes bietet ders, Geschichte der Bürokratie in Deutschland (Frankfurt 1986).

8 Thomas Ellwein, Der Staat als Zufall und Notwendigkeit. Die jüngere Verwaltungsentwicklung in Deutschland am Beispiel Ostwestfalen-Lippe, Bd. 1: Die öffentliche Verwaltung in der Monarchie 1815-1918, Bd. 2: Die öffentliche Verwaltung im gesellschaftlichen und politischen Wandel 1919-1990 (Opladen 1993/1997), hier Bd. 1 76, 12; im folgenden zitiert: Ellwein, Staat I u. II. Vgl. zusammenfassend ders., Das Dilemma der Verwaltung. Verwaltungsstruktur und Verwaltungsreformen in Deutschland (Mannheim u. a. 1994); im folgenden zitiert: Ellwein, Dilemma. 
Ellwein hat immer wieder auf die ausgeprägte Beharrungskraft jener administrativen Strukturen verwiesen, die Anfang/Mitte des 19. Jahrhunderts in Deutschland entstanden, sich bis 1914 verfestigten und seit 1919 kaum grundlegende Veränderungen erfuhren ${ }^{9}$. Offensichtlich haben weder der enorme Aufgabenzuwachs und der sukzessive Funktionswandel noch ihre organisatorisch-personelle Expansion und interne Differenzierung die öffentlichen Verwaltungen in ihrer Substanz verändert. Die tiefen politischen Zäsuren der Jahre 1918, 1933 und 1945 überdauerten sie im Kern ebenso wie die Konjunkturen der Verwaltungskritik und die wiederholten Anläufe, den gesamten Verwaltungsapparat von Grund auf zu reformieren ${ }^{10}$.

Als Ursachen dieser ausgeprägten Beharrungskraft unter ganz unterschiedlichen politischen, gesellschaftlichen und wirtschaftlichen Rahmenbedingungen hat Ellwein - neben ihrer Professionalität - zwei Grundeigenschaften der modernen Verwaltung herausgearbeitet: ihre Heterogenität und ihre Flexibilität. Jenseits der allgemeinen Bindung an das elementare Prinzip der Gesetzlichkeit hat es die „Einheit der Verwaltung " weder in institutioneller noch in personeller Hinsicht jemals auch nur näherungsweise gegeben. Doch auch die Gesetzesbindung ist durchweg mehr Programm als Wirklichkeit geblieben. Vor allem die Trennung von Aufgaben- und Kostenverantwortung im politisch-parlamentarischen Raum hat den expandierenden Bürokratien ein Maß an Handlungsautonomie zuwachsen lassen, das mit einem instrumentellen Verwaltungsverständnis herkömmlicher Prägung überhaupt nicht in Einklang zu bringen ist.

Der immer wieder gezahlte Preis für die politische Vollzugsdelegation erschöpfte sich nicht in der kaum kontrollierbaren Vollzugsautonomie der beauftragten Verwaltungen ${ }^{11}$. Diese Handlungsautonomie prägte nachhaltig das kollektive Bewußtsein des Verwaltungspersonals und die institutionelle Struktur der Verwaltungen. Aus der Selbstwahrnehmung als eigentliche Träger alltäglicher Herrschaftsausübung hat sich im Laufe der Zeit ein überwölbendes Staatsbewußtsein entwickelt. Diese spezifische Form des Etatismus bildete schon im 19. Jahrhundert die eigentliche Klammer der expandierenden Verwaltungen. Noch verstärkt wurde deren Korpsgeist in deren oberen und mittleren Rängen durch die homogenisierenden Wirkungen der forcierten Professionalisierung und der standardisierten Rekrutierung. So fragmentiert der Öffentliche Dienst objektiv auch war und ist - sein zahlenmäßiger Umfang, sein kollektives Selbstbewußtsein und seine tatsächliche Bedeutung ließen ihn bis an die unmittelbare Gegenwart heran als „Garant(en) der derzeitigen Verwaltung“ 12 erscheinen: „Die um 1815 entwikkelte Struktur dominiert" nach wie vor, stellte Ellwein noch Mitte der 1990er

9 Ellwein, Staat I 13, 56f., 76; ders., Staat II 13, 56, 79, 89, 109, 244, 324f., 537; ders., Dilemma $44,38$.

$10 \mathrm{Vgl}$. Ellwein, Staat II 54.

11 Ellwein, Staat I $43 \mathrm{f}$.; ders., Staat II $49 \mathrm{f}$.; ders., Dilemma 46.

12 Ellwein, Dilemma 33; vgl. ders., Staat II $40 \mathrm{f}$. 
Jahre fest ${ }^{13}$; auch im Zeichen der staatlichen Wiedervereinigung "regieren“ in Deutschland „Tradition und Anpassung “14.

Die wilhelminische Verwaltungspraxis wurde - wie im frühen 19. Jahrhundert - durch eine beträchtliche „Klientelorientierung " geprägt ${ }^{15}$. Darüber hinaus förderte die wachsende Vielfalt der öffentlichen Verwaltung(en) besonders im kommunalen Bereich eine gewisse Integrationsfähigkeit der bürgerlichen Institutionen gegenüber sub- und gegenkulturellen Kräften der Industriegesellschaft, insbesondere der aufsteigenden Arbeiterbewegung. Gerade diese Abweichungen vom Bild einer monolithischen Herrschaftsverwaltung á la Max Weber sind als eine "wesentliche Ursache“ für die „Kontinuität des deutschen Verwaltungsstaates" unter obrigkeitsstaatlichen, demokratischen und totalitären Auspizien benannt worden ${ }^{16}$.

Aus einer solchen Perspektive erscheint die Verwaltungskontinuität der Revolutionszeit 1918/19 geradezu selbstverständlich ${ }^{17}$. Tatsächlich wurde der Verwaltungsalltag in dieser politischen Umbruchphase weithin von dem allseitigen Bemühen geprägt, einen „Schein der Normalität" aufrecht zu erhalten. Äußerlich unbeeindruckt versahen die öffentlich Bediensteten ihren gewohnten Dienst. Die neuen Autoritäten ließen ihnen dabei zumeist freie Hand ${ }^{18}$. Während der Zusammenbruchs- und Demobilmachungskrise erwies sich die obrigkeitsstaatliche Administration weithin als kompetent, flexibel und leistungsfähig. Offenkundig „unentbehrlich und dauerhaft" stand sie nach eigenem Selbstverständnis mehr denn je „für den Staat". Allerdings hatten die Verwaltungseliten dabei mitnichten den pluralistisch-demokratischen Parteien- und Verbändestaat von Weimar im Sinn. Hinter der Fassade professioneller Pflichterfüllung blieb die übergroße Mehrheit innerlich jener „Lebenslüge des Obrigkeitsstaates“ verhaftet, die Gustav Radbruch 1930 vehement kritisierte ${ }^{19}$. Eher noch bestärkt von den zaghaften Ansätzen republikanischer Personalpolitik entwickelten sich die Fiktionen des autoritären Rechts- und Verwaltungsstaates vorrevolutionärer Zeiten und seiner überpar-

13 Ellwein, Dilemma 52; vgl. ebd. 20, 80; vgl. ferner ders., Staat I 12, 446.

14 Ellwein, Staat I 69; ders., Dilemma 58.

15 Ellwein, Staat I 470 ff.; ders., Staat II 26. Vgl. dazu nunmehr aus komparativer Perspektive Árpád von Klimó, Staat und Klientel im 19. Jahrhundert. Administrative Eliten in Italien und Preußen im Vergleich 1860-1918 (Vierow b. Greifswald 1997); ders., Politiker und Beamte in Italien und Deutschland (1860-1930). Vergleichende Überlegungen, in: Parteien im Wandel vom Kaiserreich zur Weimarer Republik. Rekrutierung - Qualifizierung - Karrieren, hrsg. von Dieter Dowe u. a. (München 1999) 341-362; ders., Zwischen Zentralstaat und Peripherie. Spitzenbeamte in Italien und Preußen-Deutschland 1870-1914, in: Zentralismus und Föderalismus im 19. und 20. Jahrhundert. Deutschland und Italien im Vergleich, hrsg. von Oliver Janz u.a. (Berlin 2000) 91-133; Florian Lang, Die Verwaltungselite in Deutschland und Frankreich 1871-2000. Regimewechsel und Pfadabhängigkeiten (Baden-Baden 2005).

$16 \mathrm{Vgl}$. Ellwein, Staat I 456, $473 \mathrm{f}$.

17 Vgl. Ellwein, Staat II 56.

18 Vgl. Ellwein, Staat II $54 \mathrm{f}$.; vgl. ebd. $537 \mathrm{f}$.

19 Gustav Radbruch, Die politischen Parteien im System des deutschen Verfassungsrechts, in: Handbuch des Deutschen Staatsrechts, hrsg. von Gerbard Anschütz, Richard Thoma (Tübingen 1930) Bd. 1285-294, hier 289. 
teilichen, allein dem Gemeinwohl verpflichteten Beamtenschaft je länger desto mehr zum imaginären Fluchtpunkt der technokratischen Tagträume des (leitenden) Verwaltungspersonals ${ }^{20}$.

Diese vordemokratischen Verhaltensdispositionen kamen Hitler und seinem Regime zugute, als sie 1933 nach den Schalthebeln der Staatsmacht griffen ${ }^{21}$. Die Staatsdiener zeigten sich fast ausnahmslos kooperationswillig, und viele von ihnen begaben sich demonstrativ in das Lager der „Nationalen Revolution“. Ansonsten arbeiteten sie abermals im Rahmen der überkommenen Strukturen, „Verfahren und Gewohnheiten“" weiter ${ }^{22}$. Nicht viel anders als 1918/19 verhielt sich auch das werdende NS-Regime. Zwar schuf es sich mit dem sogenannten „Berufsbeamtengesetz" vom April 1933 ein pseudo-legales Instrument zur Einschüchterung des Öffentlichen Dienstes. Höchste Priorität genoß aber die möglichst reibungslose Indienstnahme des hochkomplexen Verwaltungsapparates. Da ihm kaum NS-Ersatzleute zur Verfügung standen, entschieden sich Hitler und seine leitenden Gefolgsleute für ein Arrangement mit den „Professionals“23. Allen persönlichen Aversionen zum Trotz ließ die NS-Führung nicht nur Militärs und Industrielle, sondern auch die alten Funktionseliten im Bereich der Verwaltung weiterarbei$\operatorname{ten}^{24}$.

Deren übergroße Mehrzahl schlug sich mit politischer Mindestanpassung und uneingeschränkter dienstlicher Loyalität recht gut durch das Dickicht der NS-Polykratie. Ihr kollektives Selbstbehauptungslavieren trug wohl auch mit dazu bei, daß der totalitäre Zugriff des wuchernden NS- „Maßnahmenstaates“25 auf die deutsche Gesellschaft mancherlei Nischen nicht erreichte. Der zweite demokratische Versuch auf deutschem Boden sollte hernach davon profitierten. Doch zunächst wurde mit diesem Anpassungsverhalten ein essentieller Beitrag zur alltäglichen Funktionsfähigkeit der NS-Unrechtsherrschaft geleistet ${ }^{26}$. Mit Blick auf die jahrzehntelange Verdrängung dieser „Mitschuld“ der administrativen Eliten hat Thomas Ellwein Ende der 1990er Jahren treffend angemerkt: „Die Traditionsdebatte, welche die Bundeswehr immer wieder erregt hat, könnte auch in der Verwaltung geführt werden." 27

$20 \mathrm{Vgl}$. Ellwein, Staat II 11 f., 30, 56ff., 101, 281. Vgl. dazu nunmehr für Vieles Rainer Fattmann, Bildungsbürger in der Defensive. Die akademische Beamtenschaft und der „Reichsbund der höheren Beamten “ in der Weimarer Republik (Göttingen 2001).

$21 \mathrm{Vgl}$. Ellwein, Staat II 89.

22 Ebd. 89; vgl. ebd. 79, 87.

23 Ebd. 268f.; vgl. ebd. 79, 89.

$24 \mathrm{Vgl}$. ebd. $86 \mathrm{f.}, 301$.

25 Ernst Fraenkel, Der Doppelstaat. Recht und Justiz im Nationalsozialismus (Frankfurt 21984, amerik. Originalausgabe 1941).

26 Vgl. Ellwein, Staat II 299; vgl. ferner ebd. 101 f., 320.

27 Ebd. 100; vgl. ebd. 87. 


\section{Kontinuität und Transformation der westdeutschen Verwaltung in der zweiten Nachkriegszeit}

Bekanntlich sind großangelegte Personalrevirements aus Anlaß politischer Regimewechsel kein hervorstechendes Merkmal der jüngeren deutschen Verwaltungsgeschichte. Das hohe Maß institutionellen "Beharrungsvermögen(s)“ ist nicht zuletzt auf diese Tatsache zurückzuführen ${ }^{28}$. Das gilt auch für die zweite Nachkriegszeit in Westdeutschland. Anders als 1918/19 schien zwar dieses Mal unter der Ägide der Besatzungsautoritäten eine tiefgreifende Zäsur in der deutschen Verwaltungsentwicklung möglich - und zwar personell wie strukturell. Vor dem Hintergrund des wieder eskalierenden Ost-West-Konfliktes gelangten jedoch unter dem Druck der wirtschaftlichen und gesellschaftlichen Alltagsprobleme rasch wieder organisatorisch erfahrene Seniorbeamte und jüngere Technokraten in administrative Schlüsselpositionen des westdeutschen Wiederaufbaus. Der militärische und politische Zusammenbruch des NS-Regimes unterbrach mithin - wenn überhaupt - die Traditionslinie der deutschen Verwaltung im 20. Jahrhundert nur für eine kurze Übergangsphase.

Auf staatlicher wie auf kommunaler Ebene wirkte die notorische Selbstbehauptungskraft der traditionellen Verwaltung personell und institutionell bis weit in die Nachkriegszeit hinein. In den Westzonen bescherte die Phase der politischen Säuberung von 1945/46 bis 1948/49 den diskreditierten Verwaltungseliten mitnichten jene „personelle Zäsur", von der selbst in der neueren Literatur gelegentlich noch die Rede ist ${ }^{29}$. So schmerzhaft die temporären Verunsicherungen dieser Übergangszeit von den betroffenen Beamten persönlich empfunden worden sein mögen - aus der Perspektive der 1950er/60er Jahre wirkten die vielkritisierten Prozeduren der Entnazifizierung eher stabilisierend auf den Öffentlichen Dienst traditioneller Prägung. Lutz Niethammer hat die politische Säuberung treffend als „Mitläuferfabrik" und als „Schule der Anpassung“ zugleich charakterisiert ${ }^{30}: \operatorname{Im}$

28 Ellwein, Staat I 56. Zum Folgenden vgl. (mit weiteren Hinweisen) Michael Ruck, Kontinuität und Wandel - Westdeutsche Verwaltungseliten unter dem NS-Regime und in der alten Bundesrepublik, in: Verwandlungspolitik. NS-Eliten in der westdeutschen Nachkriegsgesellschaft, hrsg. von Wilfried Loth, Bernd-A. Rusinek (Frankfurt, New York 1998) 117-142; ders., Verwaltungsjuristen in der Nachkriegszeit - Wandlungen einer deutschen Funktionselite im interregionalen Vergleich, in: Themen juristischer Zeitgeschichte (3), hrsg. von Franz-Josef Düwell, Thomas Vormbaum (Baden-Baden 1999) 71-98.

29 Thomas Klein, Leitende Beamte der, allgemeinen Verwaltung in der preußischen Provinz Hessen-Nassau und in Waldeck 1867-1945 (Darmstadt, Marburg 1988) 9; vgl. ebenso Agnes Blänsdorf, Zur Konfrontation mit der NS-Vergangenheit in der Bundesrepublik, der DDR und Österreich. Entnazifizierung und Wiedergutmachungen, in: Aus Politik und Zeitgeschichte. Beilage zur Wochenzeitung Das Parlament, Nr. B 16-17/87, 18. 4. 1987, 3-18, hier 9. 30 Lutz Niethammer, Die Mitläuferfabrik. Die Entnazifizierung am Beispiel Bayerns (Berlin, Bonn 21982; zuerst Frankfurt 1972); ders., Schule der Anpassung. Die Entnazifizierung in den vier Besatzungszonen (1995), in: ders., Deutschland danach. Postfaschistische Gesellschaft und nationales Gedächtnis, hrsg. von Ulrich Herbert, Dirk van Laak u. a. (Bonn 1999) 53-58; im folgenden zitiert: Niethammer, Schule. 
Blick zurück wurden die individuellen und gruppenspezifischen Verwicklungen in die Unrechtspolitik des NS-Maßnahmenstaates nachhaltig bagatellisiert; im Blick nach vorn internalisierten die Verwaltungsleute frühzeitig die grundlegenden Normen und Verhaltensgebote jener westlichen Werte- und Verteidigungsgemeinschaft, in die sich der bundesdeutsche Teilstaat ebenso zügig wie erfolgreich eingliederte. Zusammengehalten wurde das exkulpatorisch-restaurative Bündnis von Verwaltung und Politik zum einen durch den Kalten Krieg, zum anderen durch jenen Wiederaufbaukonsens, in dessen Mittelpunkt nicht die quasi-judizielle „Bewältigung“ der NS-Vergangenheit, sondern die Überwindung der wirtschaftlichen und sozialen Alltagsprobleme stand.

Die halbherzigen Versuche der amerikanischen und britischen Besatzungsautoritäten, den Öffentlichen Dienst im allgemeinen und das Berufsbeamtentum im besonderen institutionell von Grund auf zu reformieren, scheiterten auf ganzer Linie am hinhaltenden Widerstand der Beamtenverbände und der Mitte-RechtsParteien, aber auch von Teilen der SPD und der Gewerkschaften ${ }^{31}$. Gleichzeitig kehrte in Westdeutschland das frühere Personal auf allen Ebenen wieder in die Verwaltungen zurück. Dieser personelle Restaurationsprozeß vollzog sich seit $1947 / 48$ in drei Schüben, deren Ausläufer bis in die frühen 1960er Jahre hineinreichten ${ }^{32}$. Gelegentliche Kritik prallte an dem parteienübergreifenden Einverständnis darüber $\mathrm{ab}$, daß die umfassende Reintegration der NS-Verwaltungseliten eine unabdingbare Voraussetzung für die Stabilität der jungen Bundesrepublik unter den Bedingungen des Kalten Krieges darstelle ${ }^{33}$.

31 Vgl. zuletzt Rudolf Morsey, Gefährdung und Sicherung des Berufsbeamtentums. Entwürfe und Reformkonzepte für den öffentlichen Dienst 1945-1953, in: Die Öffentliche Verwaltung 46 (1993) 1061-1070; im folgenden zitiert: Morsey, Gefährdung; vgl. ferner Hermann-Josef Rupieper, Die Wurzeln der westdeutschen Nachkriegsdemokratie. Der amerikanische Beitrag 1945-1952 (Opladen 1993) 173-204; vgl. ders., Amerikanisierung in Politik und Verwaltung Westdeutschlands. Ein problematisches Konzept, in: Amerikanisierung und Sowjetisierung in Deutschland 1945-1970, hrsg. von Konrad Jarausch, Hannes Siegrist (Frankfurt, New York 1997) 49-65; Curt Garner, Öffentlicher Dienst, in: Deutschland unter alliierter Besatzung 1945-1949/55. Ein Handbuch, hrsg. von Wolfgang Benz (Berlin 1999) 141-149; für die britische Zone vgl. ders., "Zerschlagung des Berufsbeamtentums“? Der deutsche Konflikt um die Neuordnung des öffentlichen Dienstes 1946-1948 am Beispiel Nordrhein-Westfalens, in: VfZ 39 (1991) 55-101.

32 Wolfgang Langhorst, Beamtentum und Artikel 131 des Grundgesetzes. Eine Untersuchung über Bedeutung und Auswirkung der Gesetzgebung zum Artikel 131 des Grundgesetzes unter Einbeziehung der Position der SPD zum Berufsbeamtentum (Frankfurt u.a. 1994) $153 \mathrm{ff}$. Zu den gesetzlichen Grundlagen und politischen Hintergründen der umfassenden Rückführungsaktion vgl. Udo Wengst, Beamtentum zwischen Reform und Tradition. Beamtengesetzgebung in der Gründungsphase der Bundesrepublik Deutschland 1948-1953 (Düsseldorf 1987); Norbert Frei, Vergangenheitspolitik. Die Anfänge der Bundesrepublik und die NS-Vergangenheit (München 21997) 69-100.

33 Vgl. tendenziell zustimmend Morsey, Gefährdung 1070; vgl. dagegen mit kritischem Tenor Hans Mommsen, Die Kontinuität der Institution Berufsbeamtentum und die Rekonstruktion der Demokratie in Westdeutschland, in: Zwischen Gehorsamspflicht und Widerstandsrecht: ein unlösbares Dilemma der Beamtenschaft? Dokumentation des 2. Symposiums der Dokumentations- und Forschungsstelle der FHSöV NRW für Beamten- und Ver- 
In den westdeutschen Ländern und Gemeinden hatte die personelle Rekonstruktion unter dem Druck der alltäglichen Verwaltungsprobleme der ersten Nachkriegsjahre vielfach schon vor 1947/48 eingesetzt. Denn hier trat der fundamentale Zielkonflikt zwischen raschem Wiederaufbau und personeller Säuberung der öffentlichen Dienste besonders früh und kraß zutage. Die vorrangige Sorge insbesondere der beiden angelsächsischen Siegermächte galt zusehends dem Problem, die deutschen Behörden zügig mit leistungsfähigen Fachleuten auszustatten. Dabei wurde in großem Umfang auf erprobte Verwaltungsbeamte des höheren und gehobenen Dienstes zurückgegriffen. Diese kehrten nun ihr traditionelles Leitbild eines „überparteilichen“ Fachbeamtentums wieder demonstrativ hervor. Das in seinem Ursprung vordemokratische Selbstverständnis der deutschen Bürokratie deckte sich aber nur auf den ersten Blick mit dem britischen Verständnis einer „politisch neutralen“ Verwaltung ${ }^{34}$. Dessen ungeachtet erleichterte dieses Mißverständnis es den Besatzungsautoritäten, über politische Vorbelastungen qualifizierter Kommunalbediensteter pragmatisch hinwegzusehen. Von Beginn an dominierten denn auch Angehörige der administrativen Eliten traditioneller Prägung die Kommunalverwaltungen der drei Westzonen ${ }^{35}$.

Auch in die Landesverwaltungen kehrte das noch dienstfähige Personal binnen weniger Jahre fast geschlossen zurück. Kaum jemand wurde etwa in den südwestdeutschen Innenverwaltungen von den alten Seilschaften dauerhaft ausgeschlossen, weil er sich bis 1945 politisch allzu sehr kompromittiert hatte. Schon das untergegangene NS-Regime hatte auf die Mitarbeit der traditionellen Funktionseliten nicht verzichten mögen und der zügige Neuaufbau schien - wie 1918/19 selbst Führern der Arbeiterbewegung ohne den administrativen Sachverstand der einschlägigen Fachleute ganz undenkbar. Angesichts dessen hatte etwa der größte Teil des noch dienstfähigen Personals der südwestdeutschen Innenverwaltung seine unterbrochenen Karrieren unter demokratischen Auspizien bereits wieder fortsetzen können, als die "131er"-Regelung zu greifen begann. Lediglich einige besonders stark belastete Beamte mußten sich bis in die frühen 1950er Jahre hinein gedulden, bevor auch sie auf der Basis des Grundgesetzartikels 131 wieder in den Kreis ihrer früheren Kollegen zurückkehren durften. Politisch unbelasteten

waltungsgeschichte im 20. Jahrhundert im Gebiet des heutigen Landes Nordrhein-Westfalen vom 4. und 5. Dezember 1986 in Münster, hrsg. von Friedrich G. Scbwegmann (Gelsenkirchen 1989) 65-79, hier 78f.; Niethammer, Schule.

$34 \mathrm{Vgl}$. Everhard Holtmann, Demokratische Erneuerung und nachholende Modernisierung - Deutsche Wende-Zeiten 1945 und 1989 im Vergleich, in: 1945-1995. 50 Jahre nach dem Ende des Zweiten Weltkrieges, hrsg. von Gotthard Jasper (Erlangen 1998) 37-58.

35 Vgl. dazu für Vieles als neue Referenzstudie am Beispiel von Münster in Westfalen: Sabine Mecking, "Immer treu“. Kommunalbeamte zwischen Kaiserreich und Bundesrepublik (Essen 2003); vgl. auch dies., „Besonders qualifizierte Persönlichkeit gesucht". Rekrutierungsfelder und Karrieremuster der städtischen Verwaltungselite eines typischen Behördenquartiers von 1945 bis 1975, in: Demokratisierung und gesellschaftlicher Aufbruch. Die sechziger Jahre als Wendezeit der Bundesrepublik, hrsg. von Matthias Frese u.a. (Paderborn u.a. 2003) 529-547; im folgenden zitiert: Frese u. a., Demokratisierung. Zum Folgenden vgl. Ruck, Korpsgeist 231-256. 
„Quereinsteigern“ gelang es hingegen nur in seltenen Einzelfällen, über die ersten Nachkriegsjahre hinaus in der Verwaltung Fuß zu fassen.

So folgte in Westdeutschland der Zusammenbruchskrise von 1945 bis 1947/48 unter dem Primat des „Wiederaufbaus“ zunächst ein Jahrzehnt der strukturellen wie der personellen Bewahrung und Rekonstruktion überkommener administrativer Strukturen. Und doch erlebten die Verwaltungsapparate seit den frühen 1960er Jahren einen „Modernisierungsschub“, der mit personellem Wachstum und institutioneller Differenzierung ohnegleichen einher ging. Als besonders wirkungsmächtig erwies sich dabei jener epochale Generationenwechsel in den öffentlichen Verwaltungen, welcher mit dem Mitte der 1960er Jahre verstärkt einsetzenden Wertewandel innerhalb der deutschen Gesellschaft zeitlich zusammenfiel ${ }^{36}$. Im übrigen gewann der administrative Veränderungsschub der „langen“ 1960er Jahre seine Dynamik nicht zuletzt aus jener wohlfahrtsstaatlichen Expansion, als deren Protagonisten staatliche und kommunale Verwaltungen nun verstärkt auf den Plan traten. Vor allem die jüngeren Jahrgänge der administrativen Eliten hatten mittlerweile fest jenes Leitbild des „Daseinsvorsorgestaates“ verinnerlicht, mit dem Ernst Forsthoff Ende der 1930er Jahre - erfolglos - versuchte hatte, der öffentlichen Verwaltung neuen Rückhalt im polykratischen Machtgerangel des NS-Staates zu geben ${ }^{37}$. Als der Kalte Krieg 1961/62 seine Klimax überschritten hatte, konzentrierte sich die nachrückende Generation auf die bürokratisch-technokratische Prävention und Bearbeitung innerer Konflikte im Zeichen umfassender Daseinsvorsorge.

Ein immer dichter geknüpftes Netz vertikaler und horizontaler „Fachbruderschaften " 38 zwischen den verschiedenen Verwaltungsebenen, den sozialen Selbstverwaltungskörperschaften, einschlägig interessierten Verbänden sowie ihren Vertrauensleuten in Parteien und Parlamenten verlieh den steigenden Anforderungen der Sozialadministration - im weit verstandenen Sinne des Wortes - erhebliche Durchschlagskraft im politischen Raum.

Die ökonomistische Problemwahrnehmung und die technokratischen Verhaltensdispositionen dieser jüngeren Funktionseliten legten eine Reform der Verwaltungsstrukturen nahe, die in erster Linie auf eine effizientere Ressourcenallokation abzielte. Unter den Auspizien der formal hochzentralisierten NS-Herrschaft hatte es zeitweise so ausgesehen, als könnten solche technokratischen Planungs-

36 Vgl. die Problemskizze von Michael Ruck, Verwaltung und Bürger: Einführung, in: Frese u. a., Demokratisierung 495-504.

37 Ernst Forsthoff, Rechtsfragen der leistenden Verwaltung (Stuttgart 1959); vgl. ursprünglich ders., Die Verwaltung als Leistungsträger (Stuttgart, Berlin 1938). Für die frühe Kritik an diesem Leitbild vgl. etwa Heinz-Dietrich Ortlieb, Glanz und Elend des deutschen Wirtschaftswunders, in: Bestandsaufnahme. Eine deutsche Bilanz 1962. Sechsunddreißig Beiträge deutscher Wissenschaftler, Schriftsteller und Publizisten, hrsg. von Hans Werner Richter (München u. a. 1962) 275-291.

38 Frido Wagener, Der öffentliche Dienst im Staat der Gegenwart, in: Erbard Denninger u. a., Verfassungstreue und Schutz der Verfassung. Der öffentliche Dienst im Staat der Gegenwart. Berichte und Diskussionen auf der Tagung der Vereinigung der Deutschen Staatsrechtslehrer in Bonn vom 4.-7. Oktober 1978 (Berlin, New York 1979) 215-266, hier 238ff., 251, 253. 
strategien ungehemmt Geltung erlangen, nachdem sie sich zuvor unter demokratisch-pluralistischen Verhältnissen $\mathrm{Mal}$ um $\mathrm{Mal}$ am ausgeprägten Eigensinn von Kommunen, Verbänden und Bevölkerung gebrochen hatten. Dessen parochiale Beharrungskraft ließ während der zweiten Nachkriegszeit wiederum manche Planungsbefürworter beinahe resignieren. Erst im Zeichen der neuen Aufgeschlossenheit für struktur- und prozeßgestaltende Interventionen öffentlicher Körperschaften, die sich um 1960 in der Bundesrepublik wie in anderen westlichen Industrieländern auszubreiten begann, fanden sie rasch wachsenden Rückhalt. Ihr Reformeifer kulminierte in dem Ruf nach einem grundlegenden „Neubau der Verwaltung " in funktionaler wie in territorialer Hinsicht ${ }^{39}$ - dem alsbald Proteste von Bürgerinitiativen und Forderungen nach unmittelbarer bürgerlicher Teilhabe an den administrativen Planungs- und Vollzugsprozessen entgegen schollen ${ }^{40}$.

\section{Die SBZ/DDR - ein administrativer Sonderweg}

Die administrative Bewältigung der Wiedervereinigung bestätigt im wesentlichen die skizzierte Wirkungsmächtigkeit traditioneller Verwaltungsstrukturen in Deutschland. Demgegenüber stellt sich die 1989/90 abrupt beendete Entwicklung in der SBZ/DDR im säkularen Zusammenhang als ein historischer Sonderweg dar. Durch externe Einwirkungen erzwungen, wurde das Phänomen „sozialistische Kaderverwaltung " mit deren Fortfall obsolet. Der deutsch-deutsche Einigungsvertrag von 1990 legte im Grundsatz fest, daß an ihre Stelle alsbald das westdeutsche Verwaltungsmodell zu treten habe. Dieses traditionelle Paradigma ist das Produkt eines jahrzehntelangen Prozesses der sukzessiven und retardierten Anpassung traditioneller Strukturen an die veränderten Bedingungen der Verwaltungsumwelt nach dem Verlaufsmuster: „Beharrung im Wandel“. Allerdings war dieses gradualistische Transformationsmodell auf dem Territorium der untergegangenen DDR von vornherein so nicht reproduzierbar. Das totalitäre Zwischenspiel hat dort entschieden zu lange gedauert, als daß noch unmittelbar an die administrativen Traditionsstränge aus der Zeit vor 1945 beziehungsweise 1933 hätte angeknüpft werden können ${ }^{41}$. Jedenfalls gilt das für die personalen Träger der

39 Frido Wagener, Neubau der Verwaltung. Gliederung der öffentlichen Aufgaben und ihrer Träger nach Effektivität und Integrationswert (Berlin 1969; unveränd. Nachdr. 1974).

40 Vgl. neuerdings Dieter Grunow, Hildegard Pamme, Kommunale Verwaltung: Gestaltungsspielräume und Ausbau von Partizipationschancen?, in: Frese u.a., Demokratisierung 505-528; vgl. ferner (mit weiteren Hinweisen) Michael Ruck, Ein kurzer Sommer der konkreten Utopic - Zur westdeutschen Planungsgeschichte der langen 60er Jahre, in: Dynamische Zeiten. Die 60er Jahre in den beiden deutschen Staaten, hrsg. von Axel Scbildt u.a. (Hamburg 22003; zuerst 2000) 362-401; ders., Westdeutsche Planungsdiskurse und Planungspraxis der 1960er Jahre im internationalen Kontext, in: Aufbruch in die Zukunft. Die 1960 er Jahre zwischen Planungseuphorie und kulturellem Wandel, hrsg. von Heinz-Gerhard Haupt, Jörg Requate (Weilerswist 2004) 289-325.

41 Vgl. dazu Thomas Ellwein, Tradition - Anpassung - Reform. Über die Besonderheit der Verwaltungsentwicklung in den neuen Bundesländern, in: Verwaltungsreform und Verwal- 
Verwaltungskontinuität. Der partielle Austausch administrativer Eliten und deren politische wie gesellschaftliche Degradierung hatte im Laufe der 1950er Jahre den massenhaften Exodus gelernter Verwaltungsleute herkömmlicher Prägung nach Westen bewirkt. In der DDR wurde dadurch ein vorgezogener Generationenwechsel erzwungen und ermöglicht. Unter den Begünstigten dieses Mobilitätsschubes waren viele, deren Herkunft und Ausbildung sie unter „normalen“ Umständen schwerlich in gehobene Verwaltungspositionen hätte gelangen lassen. Manches spricht dafür, daß sich unter diesen um 1920 und im folgenden Jahrzehnt geborenen Angehörigen der ostdeutschen „Aufbaugeneration“ ein vergleichsweise hohes Maß an langfristig stabilen Loyalitäten gegenüber dem SED-Regime und seiner Verwaltungsdoktrin entwickelt hat ${ }^{42}$. Freilich wurden diese Verhaltensdispositionen mit der wachsenden Enttäuschung jener nachfolgenden Generationen erkauft, deren Aufstiegserwartungen während der 1970er/80er Jahre nicht nur an der mangelnden Leistungsfähigkeit und Innovationskraft des politischen wie des ökonomischen Systems der DDR, sondern auch an der Blockade vieler Aufstiegsposten durch die Aufbaugeneration zerbrachen. Zunächst jedoch begünstigten sie mutmaßlich einen ebenso durchgreifenden wie nachhaltigen Bruch mit den institutionellen und mentalen Traditionen des deutschen Berufsbeamtentums. Mit der (und für die) Dauer des SED-Staates gewann dieser Kontinuitätsbruch - zumal unter den hermetischen Bedingungen nach dem Mauerbau den irreversiblen Charakter einer epochalen Zäsur.

\section{Fazit und Ausblick}

Im Laufe des 20. Jahrhunderts ist wiederholt versucht worden, das administrative System in Deutschland von Grund auf zu reformieren. Demgegenüber erwies(en) sich die traditionellen Verwaltung(en) durchweg als höchst unempfindlich gegenüber planvollen Modernisierungsversuchen. Aller bisherigen Erfahrung nach sind Reformen nicht durch revolutionäre Umstrukturierungen, sondern jeweils nur durch graduelle Erweiterung, Ausdifferenzierung und Modifikation der bestehenden Institutionen durchzusetzen.

Gleichwohl hat die Verwaltung ihr Gesicht im Laufe des vergangenen Jahrhunderts stark verändert - strukturell wie personell ${ }^{43}$. Dieser säkulare Transformationsprozeß resultierte nicht so sehr aus kurz- und mittelfristigen Interventionen

tungspolitik im Prozeß der deutschen Einigung, hrsg. von Wolfgang Seibel u.a. (Baden-Baden 1993) 30-40; im folgenden zitiert: Seibel u. a., Verwaltungsreform.

42 Ein Kenner der DDR-Geschichte wie Gert-Joachim Glaeßner hält diese ,tradierte(n) Verhaltensnormen und Wahrnehmungsmuster" nach wie vor für virulent; siehe ders., Vom "demokratischen Zentralismus" zur demokratischen Verwaltung? Probleme des Umbaus einer Kaderverwaltung, in: Seibel u. a., Verwaltungsreform 67-79, hier 79; vgl. Hellmut Wollmann, Wolfgang Jaedicke, Neubau der Kommunalverwaltung in Ostdeutschland - zwischen Kontinuität und Umbruch, in: ebd. 98-116, hier 106.

$43 \mathrm{Vgl}$. Ellwein, Staat II 537. 
aus dem politischen Raum, sondern aus dem sozialen Wandel, dem Wertewandel und dem politisch-konstitutionellen Wandel ${ }^{44}$.

Diese Entwicklung ist nicht kontinuierlich, sondern in zwei großen Schüben vonstatten gegangen: der eine setzte im Sommer 1914 ein, der andere zu Beginn der 1950er Jahre. Die administrativen Erfordernisse des Ersten Weltkrieges leiteten jene „zunehmende Zentralisierung von Politik und Verwaltung und die immer weiter reichende Einbindung der kommunalen Selbstverwaltung in staatliche Vollzüge" ein ${ }^{45}$, welche sich nach der Niederlage im Zeichen des Weimarer Sozialstaatskompromisses ungebrochen fortsetzte. Der zweite Veränderungsschub begann während der Rekonstruktionsphase nach dem Zweiten Weltkrieg. Unter den Bedingungen von wirtschaftlicher Prosperität, sozialstaatlicher Expansion und "nahezu revolutionäre(n)" Wandlungen der westdeutschen Gesellschaft setzte ein "grundlegender Organisationswandel" ein ${ }^{46}$.

Erst ein Vierteljahrhundert nach Gründung der Bundesrepublik bahnte sich jene "deutliche Zäsur“ innerhalb der öffentlichen Verwaltung an, die 1945 bis 1950 selbst in Ansätzen ausgeblieben war ${ }^{47}$. Bedingung und Konsequenz dieses beschleunigten Transformationsprozesses war der Verlust jener relativen Autonomie, welche die administrativen Eliten auf dem Feld ihrer personellen Selbstrekrutierung bis weit in die NS-Zeit hinein und darüber hinaus zu bewahren vermocht hatten. Diese Kooptationskraft ist seither nachhaltig durch langfristige Wandlungsprozesse geschwächt worden. Sie haben ihren Ausgang auf der Ebene der Sozialstruktur genommen und sich im Bildungssystem fortgesetzt, um schließlich in einen tiefgreifenden Wertewandel einzumünden. Politische Interventionen der NS-Machthaber oder der Besatzungsautoritäten mögen diesen Erosionsprozeß beschleunigt haben, indem sie mancherlei überkommene Strukturen beschädigten und deren Rekonstruktion verzögerten. Grundsätzlich verlief dieser Prozeß jedoch weitgehend eigengesetzlich durch die politischen Regime hindurch. Nur einmal mehr wurde deutlich, wie gering die tatsächlichen Einflußmöglichkeiten der jeweiligen politischen Eliten auf den säkularen Wandel der sozialen Strukturen im allgemeinen und auf die Rekrutierung der administrativen wie der juristischen Funktionseliten im besonderen sind.

Allen Wandlungen zum Trotz haben Thomas Ellwein und Ralf Zoll Ende der 1960er Jahre "neben wesentlichen Auflösungstendenzen ... auch noch bedeutsame Beharrungsmomente vor allem im Bereich unpolitischer Einstellungen" der

44 Ebd. 38 f.

45 Ebd. 53, 538f.

46 Ebd. 325 f., $539 \mathrm{ff}$.

47 Thomas Ellwein, Verfassung und Verwaltung, in: Zäsuren nach 1945. Essays zur Periodisierung der deutschen Nachkriegsgeschichte, hrsg. von Martin Broszat (München 1983) 4762, hier 48, 58; vgl. dazu nunmehr auch Werner Jann, Zur Entwicklung der öffentlichen Verwaltung, in: 50 Jahre Bundesrepublik Deutschland. Rahmenbedingungen - Entwicklungen Perspektiven, hrsg. von Thomas Ellwein, Everhard Holtmann (Wiesbaden, Opladen 1999) 520-543; Edwin Czerwick, Demokratisierung der öffentlichen Verwaltung in Deutschland. Von Weimar zur Bundesrepublik, in: GG 28 (2002) 183-203. 
höheren Ministerialbeamtenschaft diagnostiziert ${ }^{48}$. Das Prinzip der „demokratischen Verwaltung" britischer Provenienz hatte eben während der Besatzungszeit keine nachhaltige Resonanz unter den westdeutschen Verwaltungseliten gefunden. Mit Blick auf die 1970er/80er Jahre ist Horst Bosetzky denn auch zu dem nüchternen Befund gelangt: „Mit der ... 4-A-Strategie (Ausblendung, Abstoßung, Absorption und Adaption) ist es der Bürokratie ... gelungen, erfolgreich mit dem Phänomen Wertewandel umzugehen. "49 Andererseits hat Hans-Ulrich Derlien den oberen Rängen des deutschen Berufsbeamtentums Anfang der 1990er Jahre ausdrücklich ein hohes $\mathrm{Maß}$ an grundsätzlicher "Systemkonformität" und an „politische(r) Loyalität auch nach Regierungswechseln“ sowie eine „geringe Neigung zu technokratischen Attitüden" bescheinigt ${ }^{50}$. Sowohl die Feststellungen Bärbel Steinkempers von 1974 als auch die Ergebnisse neuester Elitenstudien weisen in die gleiche Richtung ${ }^{51}$. Freilich war und ist der etatistische Regelungsanspruch im Sinne umfassender Daseinsvorsorge ungeachtet dieser gewissen Liberalisierung in den öffentlichen Verwaltungen noch allenthalben spürbar. Erst die Zukunft wird mithin zeigen, ob der demokratisch-pluralistische Lernprozeß der administrativen Eliten in Deutschland lediglich konstellationsgebunden war -

48 Thomas Ellwein, Ralf Zoll, Berufsbeamtentum - Anspruch und Wirklichkeit. Zur Entwicklung und Problematik des öffentlichen Dienstes (Düsseldorf 1973) 206. Die Aussagen beziehen sich auf Erhebungsdaten von 1966/68.

49 Horst Bosetzky, Bürokratische Sozialisation in den Zeiten des Wertewandels, in: Systemrationalität und Partialinteresse. Festschrift für Renate Mayntz, hrsg. von Hans-Ulrich Derlien u. a. (Baden-Baden 1994) 99-122, hier 114.

50 Hans-Ulrich Derlien, Regierungswechsel, Regimewechsel und Zusammensetzung der politisch-administrativen Elite, in: Die alte Bundesrepublik. Kontinuität und Wandel, hrsg. von Bernhard Blanke, Hellmut Wollmann (Opladen 1991) 253-270, hier 268; vgl. ders., Die administrative Elite. Kontinuität und Wandel 1949 bis 1984, in: Eliten in der Bundesrepublik Deutschland, hrsg. von Landeszentrale für politische Bildung Baden-Württemberg, HansGeorg Webling (Red.), (Stuttgart 1990) 98-108; ders., Verwaltung zwischen Berufsbeamtentum und Parteipolitik: Personalrekrutierung und Personalpatronage im öffentlichen Dienst, in: Politische Bildung 21 (1988) H. 2, 57-72; ders., Continuity and Change in the West German Federal Executive Elite, 1949-1984, in: European Journal of Political Research 18 (1990) 349-372; ders., Historical Legacy and Recent Developments in the German Higher Civil Service, in: International Review of Administrative Sciences 57 (1991), 385-401; vgl. auch die umfang- und materialreiche, analytisch jedoch defiziente Studie von Cbristiane Dreber, Karrieren in der Bundesverwaltung. Voraussetzungen, Merkmale und Etappen von Aufstiegsprozessen im öffentlichen Dienst (Berlin 1996) $497 \mathrm{ff}$.

51 Bärbel Steinkemper, Klassische und politische Bürokraten in der Ministerialverwaltung der Bundesrepublik Deutschland. Eine Darstellung sozialstruktureller Merkmale unter dem Aspekt politischer Funktionen der Verwaltung (Köln u. a.) 1974. Vgl. etwa die große „Potsdamer Elitestudie" von 1995; zu deren Ergebnissen siehe ausführlich Wilhelm Bürklin, Hilke Rebensdorf u.a., Eliten in Deutschland. Rekrutierung und Integration (Opladen 1997). Zu den einschlägigen Forschungsergebnissen der 1980er Jahre vgl. Ursula Hoffmann-Lange, Eliten, Macht und Konflikt in der Bundesrepublik (Opladen 1992). Zum Gesamtkomplex vgl. nunmehr Everhard Holtmann, Vom „klassischen“ zum "politischen Bürokraten“? Einstellungen und Einstellungswandel im öffentlichen Dienst in Deutschland seit 1945, in: 50 Jahre Bundesrepublik Deutschland, hrsg. von Eckhard Jesse, Konrad Löw (Berlin 1999) 101120. 
oder ob jene "stillschweigende Revolution“, welche sich hinter den Fassaden struktureller Kontinuität seit den 1960er Jahren vollzogen haben soll, in den höheren Rängen der Verwaltung tatsächlich ein nachhaltig verändertes, krisenfestes „Rollenverständnis“" erzeugt hat ${ }^{52}$. Damit wäre in der Tat eine grundlegend neue Tradition der deutschen Verwaltung begründet worden.

52 So die optimistische Diagnose von Kenneth H. F. Dyson, Die westdeutsche „Parteibuch“Verwaltung. Eine Auswertung, in: Die Verwaltung 12 (1979) 129-160, hier $154 \mathrm{f} ., 158$. 